\title{
MICROSCOPIC CALCULATIONS OF QUANTUM PHASE TRANSITIONS IN FRUSTRATED MAGNETIC LATTICES
}

\author{
RAYMOND F. BISHOP \\ Department of Physics \\ University of Manchester Institute of Science and Technology (UMIST) \\ P.O. Box 88, Manchester M60 1QD, United Kingdom \\ raymond.bishop@manchester.ac.uk \\ SVEN E. KRÜGER \\ Department of Electrical Engineering \\ IESK, Cognitive Systems, Universität Magdeburg \\ PF 4120, 39016 Magdeburg, Germany
}

Received 1 December 2004

\begin{abstract}
It is nowadays acknowledged that the coupled cluster method (CCM) provides one of the most powerful and most widely used of all $a b$ initio techniques of microscopic quantum many-body theory. It has been applied to a broad range of both finite and extended physical systems defined on a spatial continuum, where it has generally yielded numerical results which are among the most accurate available. This widespread success has spurred many recent applications to quantum systems defined on a lattice. We discuss here a. typical example of a two-dimensional spin-half Heisenberg magnet with two kinds of competing nearest-neighbour bonds. We show how the CCM can successfully describe the influence of strong quantum fluctuations on the zero-temperature phases and their quantum phase transitions. The model shows how the CCM can successfully describe the effects of competition between magnetic bonds with and without the presence of frustration. The frustrated case is particulary important since many other methods, including quantum Monte Carlo simulations, typically fail in this regime.
\end{abstract}

Keywords: Coupled cluster method; quantum phase transitions; magnetic lattices; frustration; bond competition.

\section{Introduction}

The coupled cluster method $(\mathrm{CCM})^{1}$ has become one of the most pervasive and most powerful of all $a b$ initio formulations of microscopic quantum many-body theory. It has been widely applied to many diverse physical and chemical systems defined on a spatial continuum, ${ }^{2}$ where it has yielded numerical results which are usually among the best available for these systems. This widespread success has spurred recent further applications to similar strongly-correlated quantum-mechanical systems defined on an extended regular spatial lattice.

Such spin- and electron-lattice systems have become the subject of intense theoretical study. They include many examples of systems characterized by novel ground 
states which display quantum order in some region of the Hamiltonian parameter space, delimited by critical values which mark the corresponding quantum phase transitions. The critical phenomena in these quantum-mechanical models often differ profoundly from those of their classical counterparts, and the subtle correlations present usually cannot sensibly be treated by such standard many-body techniques as perturbation theory or mean-field approximations. One of the key challenges for modern quantum many-body theory is to develop and exploit microscopic techniques capable of handling both these novel and more traditional systems. Our recent work ${ }^{3}$ shows that one such method which is capable of bridging this divide is the CCM.

In particular, we have shown how the systematic inclusion of multispin correlations for a wide variety of quantum spin-lattice problems can be very efficiently implemented with the CCM. ${ }^{4}$ The method is not restricted to bipartite lattices or to non-frustrated systems, and can thus deal with problems ${ }^{3-13}$ where the quantum Monte Carlo (QMC) techniques would be faced with the infamous "minus-sign problem". We note that the method is also easily extended to models of strongly interacting electrons on lattices, such as the Hubbard model. ${ }^{14}$

In Sec. 2 we first very briefly review the CCM itself as a universal tool of quantum many-body theory. We then discuss an illustrative example from among many applications which have already been made to quantum spin-lattice systems. The model itself, which contains two types of competing nearest-neighbour interactions, is first described in Sec. 3, where its classical ground state is also discussed. The model is particularly interesting because, inter alia, it exhibits competition between magnetic order and dimerization. As in all other cases the CCM may readily be implemented for this model to high orders using computer-algebraic techniques. Results are presented in Sec. 4, where critical comparisons are made with the results from such other competing techniques as exact diagonalisations of small lattices, and (both linear and second-order renormalized) spin-wave theory. We conclude with a discussion of the results in Sec. 5 .

\section{The Coupled Cluster Method}

The starting point for any CCM calculation (and see the overviews in Refs. [1, 2] for more details) is the choice of a normalized model or reference state $|\Phi\rangle$, together with a set of mutually commuting multispin creation operators $C_{I}^{+}$which are defined over a complete set of many-body configurations $I$. The operators $C_{I}$ are the multispin destruction operators and are defined to be the Hermitian adjoints of the $C_{I}^{+}$operators. We choose $\left\{|\Phi\rangle ; C_{I}^{+}\right\}$in such a way that we have $\langle\Phi| C_{I}^{+}=0=$ $C_{I}|\Phi\rangle, \forall I \neq 0$, with $C_{0}^{+} \equiv 1$.

For spin systems an appropriate choice for the CCM model state $|\Phi\rangle$ is often a classical spin state, ${ }^{3}$ in which the most general situation is one in which each spin can point in an arbitrary direction. After a local canonical transformation on each 
site to rotate each spin to the down $(-z)$ direction, we have

$$
|\Phi\rangle=|\cdots \downarrow \downarrow \downarrow \cdots\rangle ; \quad C_{I}^{+}=s_{r}^{+}, s_{r}^{+} s_{r^{\prime}}^{+}, s_{r}^{+} s_{r^{\prime}}^{+} s_{r^{\prime \prime}}^{+}, \cdots,
$$

(where the indices $r, r^{\prime}, r^{\prime \prime}, \ldots$ denote any lattice site) respectively, for the model state and the multispin creation operators which consist of spin-raising operators only.

The CCM parameterizations of the ket and bra ground states are given by

$$
|\Psi\rangle=e^{S}|\Phi\rangle, \quad S=\sum_{I \neq 0} \mathcal{S}_{I} C_{I}^{+}, \quad\langle\tilde{\Psi}|=\langle\Phi| \tilde{S} e^{-S}, \quad \tilde{S}=1+\sum_{I \neq 0} \tilde{\mathcal{S}}_{I} C_{I} .
$$

To find the ket-state and bra-state correlation coefficients $\mathcal{S}_{I}$ and $\tilde{\mathcal{S}}_{I}$, respectively, we require that the expectation value $\bar{H}=\langle\tilde{\Psi}|H| \Psi\rangle$ be stationary with respect to variations in the bra-state and ket-state correlation coefficients. Hence, for example, the CCM ket-state equations are given by

$$
\left\langle\Phi\left|C_{I} e^{-S} H e^{S}\right| \Phi\right\rangle=0, \quad \forall I \neq 0 .
$$

The problem of determining the CCM equations now becomes a pattern-matching exercise of the $\left\{C_{I}\right\}$ to the terms in $e^{-S} H e^{S}$ in Eq. (3). The excited states may also be parametrized in a similar fashion (and see Refs. [1,3] for details).

We now use the lattice symmetries in order to find all different possible configurations with respect to the point and space group symmetries (of both the lattice and Hamiltonian) with up to $n$ spins flips spanning a range of no more than $n$ adjacent lattice sites (LSUB $n$ approximation) and these are referred to as the fundamental configurations or clusters. Details of the CCM computational algorithm for spin quantum number $s=1 / 2$ are given in Refs. [3, 9].

\section{A Typical Spin-Lattice Model Exhibiting Various Phases}

We consider a spin-half Heisenberg model on a square lattice with two kinds of nearest-neighbour bonds $J$ and $J^{\prime}$, as shown in Fig. 1,

$$
H=J \sum_{\langle i j\rangle_{1}} \mathbf{s}_{i} \cdot \mathbf{s}_{j}+J^{\prime} \sum_{\langle i j\rangle_{2}} \mathbf{s}_{i} \cdot \mathbf{s}_{j} .
$$

The expressions $\langle i j\rangle_{1}$ and $\langle i j\rangle_{2}$ indicate nearest-neighbour bonds arranged in a regular zigzag pattern, as shown in Fig. 1 by the dotted and solid lines, respectively. Each square-lattice plaquette consists of three $J$ bonds and one $J^{\prime}$ bond. If $J^{\prime}$ and $J$ have different signs then the plaquettes are frustrated, whereas competition without frustration is realized for antiferromagnetic bonds $J^{\prime}>J>0$. A model with such a zigzag pattion of bonds has been treated previously by a variety of methods. ${ }^{10,11,15,16}$

The case of antiferromagnetic $J^{\prime}$ bonds with $J^{\prime}>J>0$ resembles the situation in bilayer systems and in the depleted square-lattice antiferromagnet $\mathrm{CaV}_{4} \mathrm{O}_{9}$, in which the competition between two different antiferromagnetic bonds leads to a phase transition from antiferromagnetic long-range order to quantum disorder with 

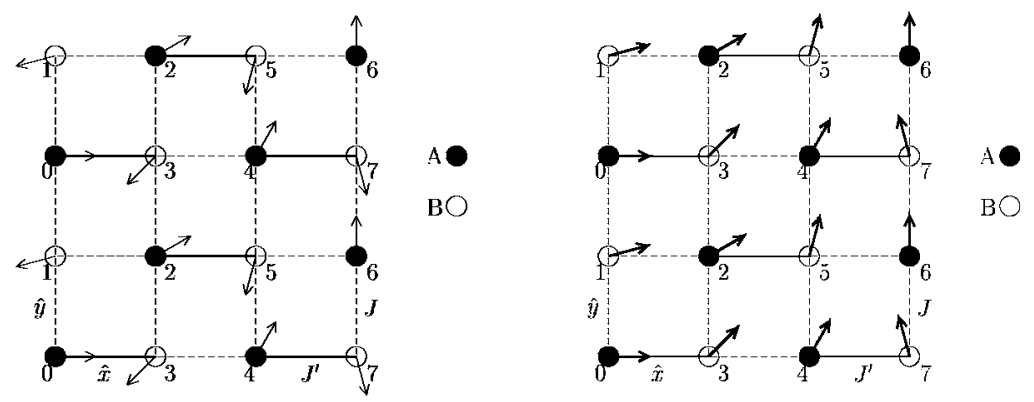

Fig. 1. Illustration of the model ( $J$-bonds correspond to dotted lines and $J^{\prime}$-bonds to solid lines) and of the classical spiral state for antiferromagnetic $J=+1$ and ferromagnetic $J^{\prime}<-1 / 3$ (left graph) and vice versa $\left(J=-1, J^{\prime}>1 / 3\right)$ (right graph). As discussed in the text, both spiral states can be transformed into each other by reversing all of the spins on the B sublattice.

a finite gap. It is seen in this article that the transition point obtained for the model of Eq. (4) is quite close to that obtained for the bilayer model. ${ }^{17}$

For special values of $J$ and $J^{\prime}$ the model represents (i) the spin-half Heisenberg antiferromagnet (ferromagnet) on the square lattice for $J=J^{\prime}=1\left(J=J^{\prime}=\right.$ -1), (ii) the spin-half Heisenberg antiferromagnet (ferromagnet) on the honeycomb lattice for $J=1, J^{\prime}=0\left(J=-1, J^{\prime}=0\right)$, and (iii) the spin-one Heisenberg antiferromagnet on the triangular lattice for $J=1, J^{\prime}=-\infty$.

\subsection{The classical ground state}

For $J=1$ and $J^{\prime}>-1 / 3$ the Néel state is the classical ground state of the Hamiltonian of Eq. (4). At $J_{c}^{\prime}=-1 / 3$ there is classically a second-order phase transition to a ground state of helical nature (see Fig. 1), with a characteristic pitch angle $\Phi= \pm\left|\Phi_{\mathrm{cl}}\right|$ given by

$$
\left|\Phi_{\mathrm{cl}}\right|= \begin{cases}0 & J^{\prime}>-\frac{1}{3} \\ \arccos \left(\frac{1}{2} \sqrt{1-\frac{1}{J^{\prime}}}\right) & J^{\prime} \leq-\frac{1}{3}\end{cases}
$$

where the different signs correspond to the two chiralities ${ }^{18}$ of this helical state. Note that for $\Phi=0$ this is just the Néel state. More generally, the pitch angle varies with $J^{\prime}$ from $\left|\Phi_{\mathrm{cl}}\right|=0$ for $J^{\prime}>-1 / 3$ to $\left|\Phi_{\mathrm{cl}}\right|=\pi / 3$ for $J^{\prime}=-\infty$. Note that $\left|\Phi_{\mathrm{cl}}\right|=\pi / 3$ (realized at $J^{\prime}=-\infty$ ) corresponds to the ground state of the spin-1 triangular lattice. For the $J=1$ case we describe the directions of the spins $\mathbf{s}_{A}$ and $\mathbf{s}_{B}$, belonging to the $A$ and $B$ sublattices respectively, for the classical helical state with a characteristic angle $\Phi$ as follows ${ }^{10}$ (and see Fig. 1),

$$
\begin{aligned}
\mathbf{s}_{A}(\mathbf{R}) & =\hat{\mathbf{u}} \cos \mathbf{Q} \cdot \mathbf{R}+\hat{\mathbf{v}} \sin \mathbf{Q} \cdot \mathbf{R}, \\
\mathbf{s}_{B}(\mathbf{R}+\hat{x}) & =\hat{\mathbf{u}} \cos (\mathbf{Q} \cdot \mathbf{R}+\pi+3 \Phi)+\hat{\mathbf{v}} \sin (\mathbf{Q} \cdot \mathbf{R}+\pi+3 \Phi),
\end{aligned}
$$


where $\hat{\mathbf{u}}$ and $\hat{\mathbf{v}}$ are perpendicular unit vectors in the spin space, $\mathbf{R}$ runs over the sites of the sublattice $A$, and we have $\mathbf{Q}=(2 \Phi, 0)$ for the pitch vector $\mathbf{Q}$. We note that this general helical state does not have a periodicity in the $x$-direction because $\Phi$ is in general not of the form $m \pi / n$ with $m$ and $n$ integral. We also note that we have only three different angles between nearest-neighbour spins, namely $\pm(\pi-\Phi)$ for the $J=1$ couplings and $\pi-3 \Phi$ for the coupling with $J^{\prime}$.

The maximum frustration is in the region around $J^{\prime} \approx-1$. Bearing in mind the situation for the $J_{1}-J_{2}$ model, one might expect that for the extreme quantum case (spin-half) quantum fluctuations might be able to open a window to a spin-liquid phase for a finite range of parameters around this region of maximum frustration. On the other hand, for strong antiferromagnetic bonds $\left(J^{\prime} \gg 1\right)$ there is, of course, no indication in the classical model for the breakdown of the Néel order.

By contrast with the quantum case, the classical model with $J=-1$ can be simply transformed into the model with $J=+1$ considered above by the simultaneous substitutions $J \rightarrow-J, J^{\prime} \rightarrow-J^{\prime}, \mathbf{s}_{i \in B} \rightarrow-\mathbf{s}_{i \in B}$. Hence, the physics for the $J=-1$ case in the classical model is essentially the same as for the $J=+1$ case (and see Fig. 1).

The resulting number of CCM LSUB $n$ configurations for $n \leq 8$ are shown in Table 1, using both the Néel and spiral states as model states.

Table 1. Number of fundamental ground-state configurations of the LSUBn approximation for the Hamiltonian of Eq. (4), using a Néel state $(\Phi=0)$ and a helical state $(\Phi \neq 0)$ for the CCM model state, and the number of fundamental excited-state configurations using the Néel model state only.

\begin{tabular}{rrrr}
\hline LSUB $n$ & ground state: $\Phi=0$ & $\Phi \neq 0$ & excited state: $\Phi=0$ \\
\hline 2 & 3 & 5 & 1 \\
4 & 22 & 76 & 16 \\
6 & 267 & 1638 & 331 \\
8 & 4986 & 42160 & 7863 \\
\hline
\end{tabular}

\section{Results}

\subsection{Antiferromagnet: Néel versus dimer phases}

In order to set the scene we start with a simple mean-field (MF) like description of the order-disorder transition. The corresponding uncorrelated MF state for Néel longe-range order (LRO) is the Néel state $\left|\phi_{\mathrm{MF}_{1}}\right\rangle=|\uparrow \downarrow \uparrow \ldots\rangle$, and for the dimerized singlet state it is the rotationally-invariant product state of local pair singlets $\left|\phi_{\mathrm{MF}_{2}}\right\rangle=\prod_{i \in A}\left[\left|\uparrow_{i} \downarrow_{i+\hat{x}}\right\rangle-\left|\downarrow_{i} \uparrow_{i+\hat{x}}\right\rangle\right] / \sqrt{2}$ where $i$ and $i+\hat{x}$ correspond to those sites which cover the $J^{\prime}$ bonds. In order to describe the transition between both states we consider an uncorrelated product state interpolating between $\left|\phi_{\mathrm{MF}_{1}}\right\rangle$ and 

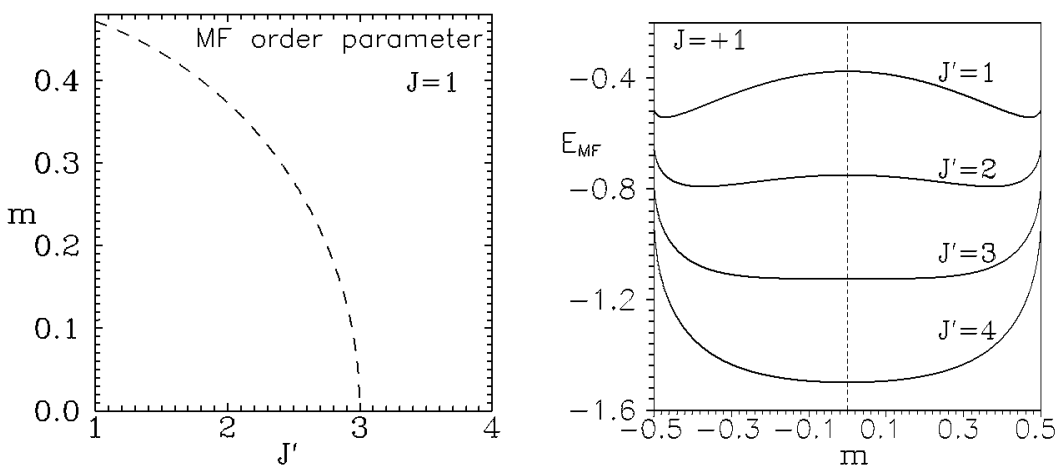

Fig. 2. Sublattice magnetization versus $J^{\prime}$ (for $J=1$ ) (left graph), and energy versus sublattice magnetization (right graph), using a mean field approach as in Eq. (7).

$\left|\phi_{\mathrm{MF}_{2}}\right\rangle$, of the form ${ }^{10,19}$

$$
\left|\Psi_{\mathrm{MF}}(t)\right\rangle=\prod_{i \in A} \frac{1}{\sqrt{1+t^{2}}}\left[\left|\uparrow_{i} \downarrow_{i+\hat{x}}\right\rangle-t\left|\downarrow_{i} \uparrow_{i+\hat{x}}\right\rangle\right] .
$$

We have $\left|\Psi_{\mathrm{MF}}(t=0)\right\rangle=\left|\phi_{\mathrm{MF}_{1}}\right\rangle$ and $\left|\Psi_{\mathrm{MF}}(t=1)\right\rangle=\left|\phi_{\mathrm{MF}_{2}}\right\rangle$. We minimize $\left\langle\Psi_{\mathrm{MF}}|H| \Psi_{\mathrm{MF}}\right\rangle$ with respect to $t$ and obtain

$$
\frac{E_{\mathrm{MF}}}{N}=\frac{\left\langle\Psi_{\mathrm{MF}}|H| \Psi_{\mathrm{MF}}\right\rangle}{N}= \begin{cases}-\frac{3 J^{\prime}}{8}-\frac{1}{24 J}\left(3 J-J^{\prime}\right)^{2} & J^{\prime} \leq 3 J \\ -\frac{3 J^{\prime}}{8} & J^{\prime}>3 J\end{cases}
$$

for the energy per site. For the sublattice magnetization, $m \equiv\left\langle\Psi_{\mathrm{MF}}\left|s_{i \in A}^{z}\right| \Psi_{\mathrm{MF}}\right\rangle$, we get $m=\sqrt{\left(3 J-J^{\prime}\right)\left(3 J+J^{\prime}\right)} /(6 J)$ for $J^{\prime} \leq 3 J$, and $m=0$ otherwise. Note that $m$ vanishes at a critical point $J_{c}^{\prime}=3 J$, and that the critical index is the MF index $1 / 2$. Equation (8) may be rewritten in terms of $m$ as $E_{\mathrm{MF}} / N=-\frac{1}{8} J^{\prime}-$ $\frac{1}{4} J^{\prime} \sqrt{1-4 m^{2}}-\frac{3}{2} J m^{2}$, and Fig. 2 illustrates that the dependence of $E_{\mathrm{MF}}$ on $m$ corresponds to a typical scenario of a second-order transition (see Ref. [12]). We can expand $E_{\mathrm{MF}}$ up to fourth order in $m$ near the critical point and find a Landau-type expression, given by $E_{\mathrm{MF}} / N=-\frac{3}{8} J^{\prime}+\frac{1}{2}\left(J^{\prime}-3 J\right) m^{2}+\frac{1}{2} J^{\prime} m^{4}$. However, as discussed elsewhere for a similar magnetic model for $\mathrm{CaV}_{4} \mathrm{O}_{9},{ }^{20} \mathrm{MF}$ theory is not expected to describe the critical behaviour correctly.

Let us therefore now apply a high-order CCM approach (for details see Refs. [3, 10]) to this model. We set the classical collinear Néel state to be the reference state $|\Phi\rangle$. We calculate the ground-state (GS) wave function, $|\Psi\rangle=e^{S}|\Phi\rangle$ within the LSUB $n$ approximation scheme up to $n=8$ and extrapolate to $n \rightarrow \infty$. The CCM results for the order parameter are shown in the left graph of Fig. 3 and they are compared to results of linear spin wave theory (SWT), the extrapolated results from exact diagonalization (ED) of $N=16,18,20,26,32$ sites, and the MF theory. The CCM is able to describe correctly the order-disorder transition, whereas conventional SWT cannot (and for more details concerning the SWT and ED results see Ref. [10]). The critical value predicted by extrapolation of the LSUB $n$ results is, 

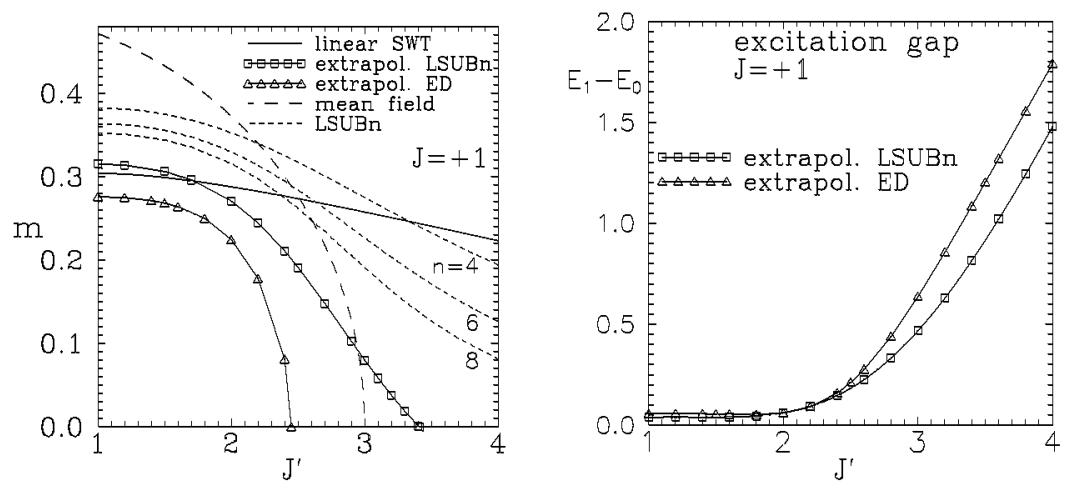

Fig. 3. Sublattice magnetization (left graph) and excitation gap (right graph) versus $J^{\prime}$.

however, found to be slightly too large. We may also consider the inflection points of $m$ versus $J^{\prime}$ for the LSUBn approximations. It is assumed that the true $m\left(J^{\prime}\right)$ curve will have a negative curvature up to the critical point. Thus we might expect that (for increasing $n$ ) the inflection point approaches the critical point. We find the corresponding inflection points at $J^{\prime}=3.1(n=2), J^{\prime}=3.0(n=4), J^{\prime}=2.9(n=6)$ and $J^{\prime}=2.85(n=8)$, indicating a critical value $J_{c}^{\prime}$ somewhere between $2.5 J$ and $3 J$. Notice that the estimation of $2.5 \lesssim J_{c}^{\prime} / J \lesssim 3$ is consistent with results of series expansions and exact diagonalizations. ${ }^{10,15}$ The breakdown of Néel LRO due to singlet formation is also accompanied by the opening of an excitation gap between the singlet GS and the first triplet excitation. This behaviour is well described by the CCM (right graph of Fig. 3) which predicts that the gap opens in the range $2 J<J^{\prime}<3 J$ (and notice that the non-zero gap below about $2 J$ is a result of the limited accuracy of the extrapolation).

\subsection{Antiferromagnet: Néel versus spiral phases}

In Sec. 4.1 we restricted our attention to the region $\left(J=+1, J^{\prime}>0\right)$ where we have "competition without frustration" between the bonds. The two terms within the Hamiltonian of Eq. (4) compete because the first term $(J)$ favours Néel longrange order on the honeycomb lattice, whereas the second term $\left(J^{\prime}\right)$ favours an uncorrelated dimerized state of local pair singlets. By contrast, we now turn our attention to the frustrated model (i.e., "competition with frustration") where $J$ and $J^{\prime}$ have opposite signs. Firstly, in this subsection we consider the case $J=+1$, $J^{\prime}<0$ where the Néel and spiral states compete, and in the next subsection consider the case $J=-1, J^{\prime}>0$ where the ferromagnetic and spiral states compete. In both cases the ED technique for finite-size lattices is less useful because of the incommensurate nature of the classical spiral states. By contrast, the CCM considers the limit $N \rightarrow \infty$ from the outset and should have no such problem dealing with incommensurate states. In both cases we use the corresponding classical state of 

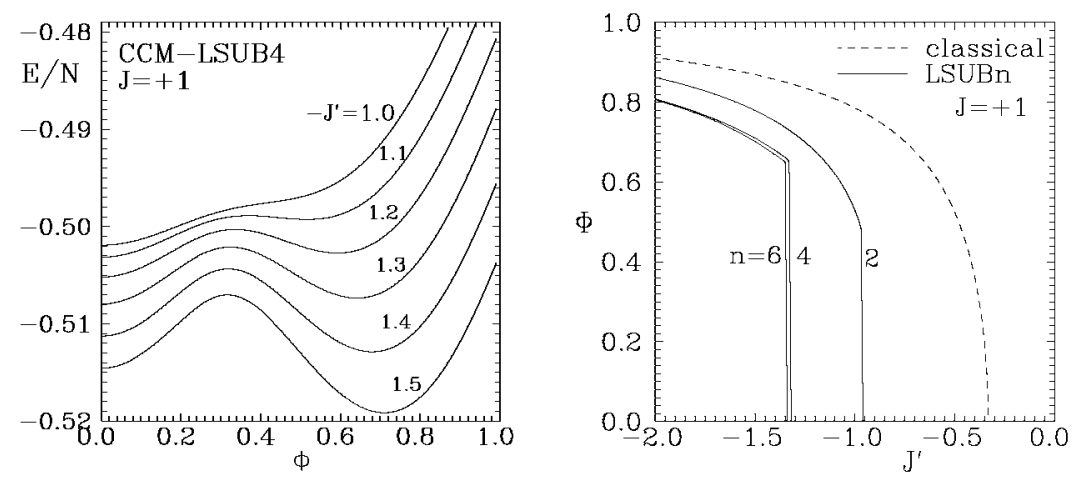

Fig. 4. Energy versus quantum pitch angle for LSUB4 (left graph) and quantum pitch angle versus $J^{\prime}$ (right graph). Note, that $\Phi=0$ corresponds to the Néel state.

Sec. 3.1 as the CCM model state. However, since quantum fluctuations can clearly change the spiral pitch angle from its classical value, we determine the "quantum pitch angle" $\Phi$ by minimizing the energy $E_{\mathrm{LSUB} n}(\Phi)$ with respect to $\Phi$ in each order $n$.

Results for $E(\Phi)$ and $\Phi\left(J^{\prime}\right)$ are shown in Fig. 4 for the case $J=+1, J^{\prime}<0$. Several key points emerge. Firstly, it is clear that the CCM continues to yield a consistent description of the system in both these collinear and spiral phases. Secondly, the quantum Néel state remains the ground state up to much stronger frustration than in the classical case. This is in keeping with the general observation that quantum fluctuations in spin systems tend to favour collinear spin structures over non-collinear ones. Thirdly, and very strikingly, we observe that in this case the quantum fluctuations also change the order of the phase transition from second order in the classical case to first order in the quantum case.

\subsection{Ferromagnet: Ferromagnetic versus spiral phases}

We now contrast the case considered in Sec. 4.2 with the case where $J=-1$, $J^{\prime}>0$. Whereas in the classical model we again have a second-order transition between collinear and spiral phases, the quantum model in these two regimes behaves quite differently. Although the collinear antiferromagnetic phase contains strong quantum fluctuations as we saw above, the fully polarized, collinear ferromagnet contains no such fluctuations. The corresponding results for $E(\Phi)$ and $\Phi\left(J^{\prime}\right)$ are displayed in Fig. 5. In this case the order of the transition from the collinear (ferromagnetic) to the noncollinear (spiral) phase remains second order, as in the classical case. Furthermore, the quantum critical point remains exactly at the classical value, $J^{\prime} / J=-1 / 3$. 

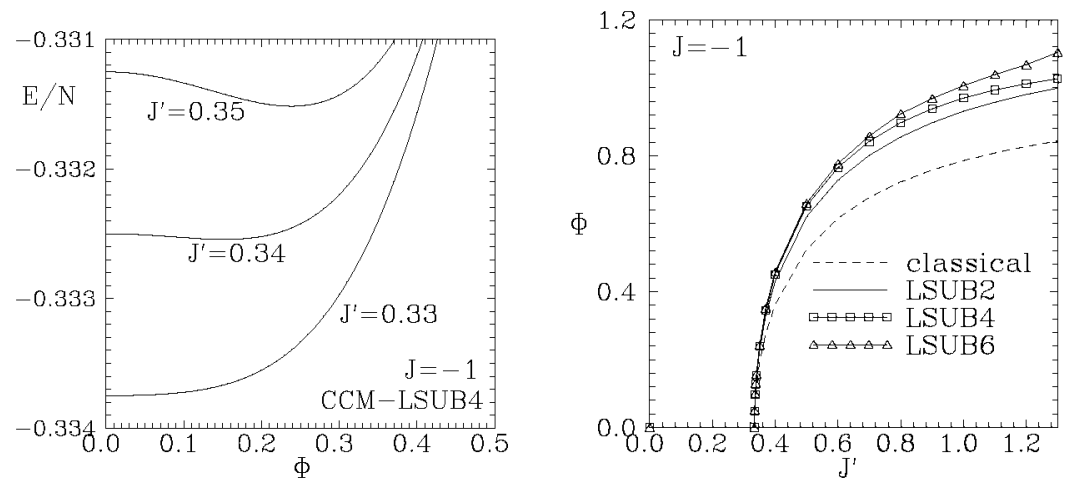

Fig. 5. Energy versus quantum pitch angle for LSUB4 (left graph) and quantum pitch angle versus $J^{\prime}$ (right graph). Note, that $\Phi=0$ corresponds to the fully polarized ferromagnetic state.

\subsection{Comparison of the various cases}

The difference between the two cases considered in Secs. 4.2 and 4.3 is particularly illustrated by Fig. 6 which contrasts the behaviour of the order parameter, namely the on-site magnetic moment $m$, in the two regimes. For $J=+1$ there exists a
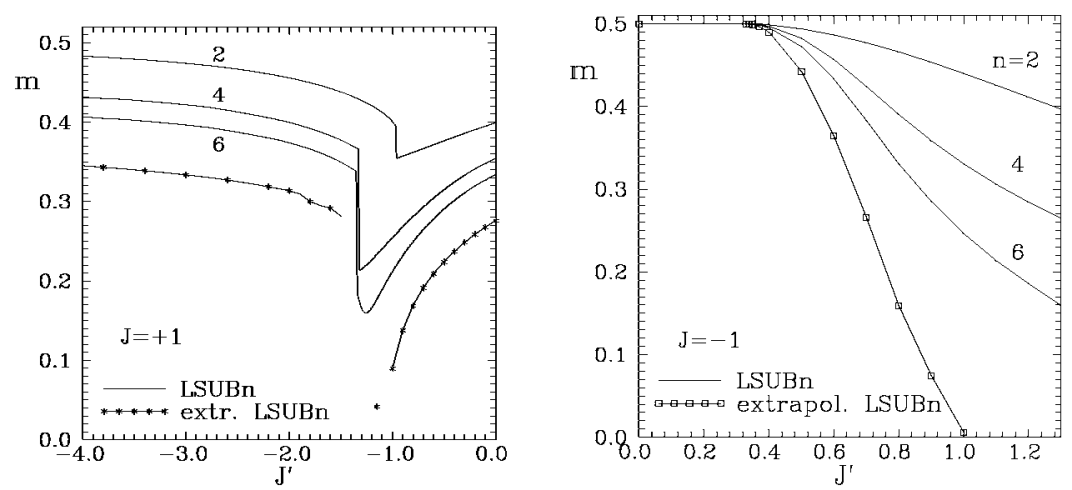

Fig. 6. On-site magnetic moment versus $J^{\prime}$ for $J=+1$ (left graph) and $J=-1$ (right graph).

discontinuity in $m$ at every level of $\operatorname{LSUB} n$ approximation. This is a reflection of the behaviour observed in $E(\Phi)$ in Fig. 4, where a local minimum at $\Phi \neq 0$ appears for $J^{\prime} \lesssim-1.1$, which for values $J^{\prime} \lesssim-1.35$ becomes a global minimum. The extrapolation of $m$ to the exact $n \rightarrow \infty$ limit does, however, become imprecise close to the phase transition point, and we cannot decide with certainty whether the order parameter does vanish at the transition point or, indeed, over a small finite regime around it. Figure 6 displays a minimum extrapolated value of $m \approx 0.05$ at $J_{c}^{\prime} \approx-1.2$. We cannot exclude entirely a disordered quantum spin-liquid phase with $m=0$ caused by quantum fluctuations plus frustration, but if it does exist it 

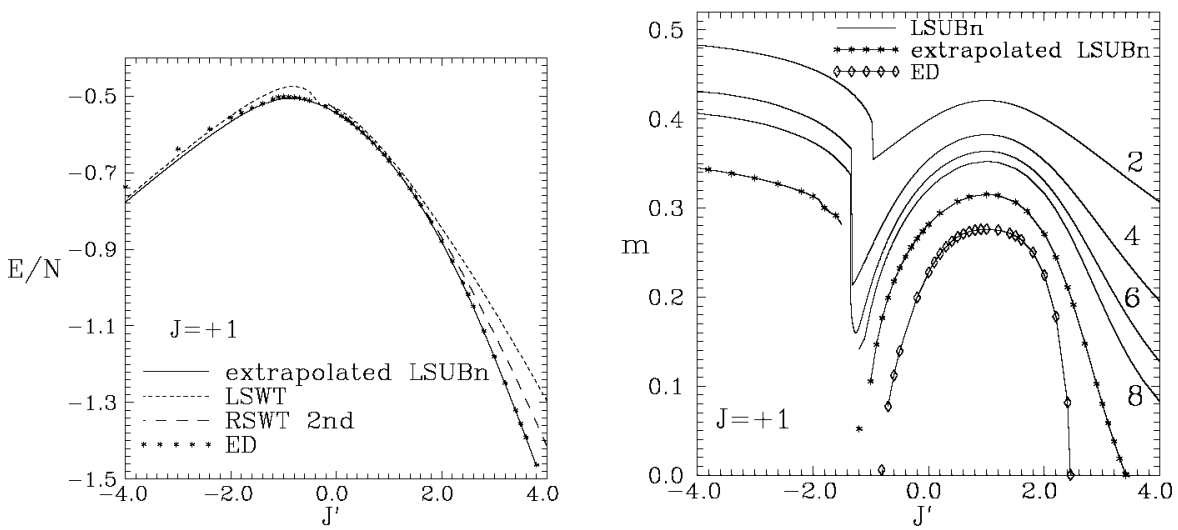

Fig. 7. Left: Ground-state energy versus $J^{\prime}$, for the case $J=+1$, for the extrapolated CCMLSUB $n$ approximations, in comparison with results of spin-wave theory (linear and second-order renormalized) ${ }^{16}$, and with the extrapolated result of exact diagonalization (ED) data. Right: Ground-state magnetic order parameter versus $J^{\prime}$, for the case $J=+1$, for the CCM-LSUBn approximation. The results are compared (for the Néel region only) with the extrapolated results of exact diagonalization (ED) data. Note that both extrapolated results fit poorly in a region around $J^{\prime} \approx-1$, and we therefore plot them here as isolated points (omitting the solid lines).

can clearly occur only over a very narrow regime in $J^{\prime}$ around $-1.5 \lesssim J^{\prime} \lesssim-1.1$. The most likely scenario however is a single phase boundary at a value $J_{c}^{\prime} \approx-1.35$ between spiral and Néel phases, with no separate spin-liquid phase.

By contrast, Fig. 6 shows a smooth change in $m$ at the critical point $J_{c}^{\prime}=1 / 3$ for $J=-1$. For increasing $J^{\prime}>J_{c}^{\prime}$ the spiral magnetic order becomes weaker and finally vanishes at $J^{\prime} \approx 1$. The underlying reason for this is again local singlet formation (i.e., dimerization), as discussed in Sec. 4.1. On the other hand, a much smaller critical strength $J^{\prime}$ is now needed for dimerization than in the case $J=+1$ (where $2.5 \lesssim J_{c}^{\prime} \lesssim 3$ ), due to the effects of frustration which now assist in the formation of local singlets. ${ }^{19}$

Finally, in Fig. 7 we show both the ground-state energy $E\left(J^{\prime}\right)$ and the order parameter $m\left(J^{\prime}\right)$ in the case $J=+1$ over the regimes spanning the spiral, Néel, and dimer-like phases. We observe excellent agreement between the CCM results and the results from exact diagonalization (ED) for $J^{\prime}>0$. By contrast, spin-wave theory (SWT) calculations ${ }^{16}$ show a significant deviation for larger $J^{\prime}>>1$. These latter are obviously poor since they lie above the simple variational upper bound of Eq. (8). The CCM and ED results, by comparison, lie slightly below the mean-field variational result.

We note that although the Néel state is the starting reference state for both CCM and SWT calculations, the CCM is much better able than SWT to describe the transition to the rotationally-invariant disordered state and hence to its limiting form, namely the fully dimerized state of Eq. ( 7 ) with $t=1$. For example, even the simplest LSUB2 CCM approximation gives the correct asymptotic behaviour for 
the ground-state energy, $E / N \rightarrow-3 J^{\prime} / 8$ as $J^{\prime} \rightarrow \infty$, whereas SWT does not.

\section{Discussion}

We have seen that the CCM works extremely well over several distinct phases of a spin-lattice model with a relatively complex phase diagram, including giving accurate predictions of the phase boundaries. By contrast, standard SWT, even in higher-order implementations, fails to describe the transition caused by the destruction of Néel longe-range order due to local singlet formation. In this region, where frustration is absent, on the other hand, other standard techniques such as QMC can be applied, since no sign problem arises here. Conversely, where we have competition with frustration, the QMC is not easily applicable. The CCM, almost alone among universal methods, yields a consistent description of collinear, noncollinear, and disordered phases. It has even been shown to be able to describe the quantum first-order transition from the Néel phase to the helical phase, for which the classical model has a second-order transition.

Based on the success of the CCM to describe this and other complex magnetic lattice models, it would be interesting to extend the method both to other strongly correlated electron-lattice models and to include the effects of finite (nonzero) temperatures. In the latter regard one may then expect to be able to use the CCM to investigate quantitatively the competing effects of quantum and thermal fluctuations in a fully microscopic formalism.

\section{References}

1. R.F. Bishop, in Microscopic Quantum Many-Body Theories and Their Applications, edited by J. Navarro and A. Polls, Lecture Notes in Physics Vol. 510 (Springer-Verlag, Berlin, 1998), p. 1.

2. R.F. Bishop, Theor. Chim. Acta 80, 95 (1991); R.J. Bartlett, J. Phys. Chem. 93, 1697 (1989).

3. C. Zeng, D.J.J. Farnell, and R.F. Bishop, J. Stat. Phys. 90, 327 (1998); D.J.J. Farnell, R.F. Bishop, and K.A. Gernoth, J. Stat. Phys. 108, 401 (2002).

4. D.J.J. Farnell and R.F. Bishop, in Quantum Magnetism, edited by U. Schollwöck, J. Richter, D.J.J. Farnell, and R.F. Bishop, Lecture Notes in Physics Vol. 645 (SpringerVerlag, Berlin, 2004), p. 307.

5. R.F. Bishop, R.G. Hale, and Y. Xian, Phys. Rev. Lett. 73, 3157 (1994).

6. D.J.J. Farnell, S.E. Krüger, and J.B. Parkinson, J. Phys.: Condens. Matter 9, 7601 (1997).

7. R.F. Bishop, D.J.J. Farnell, and J.B. Parkinson, Phys. Rev. B 58, 6394 (1998).

8. R.F. Bishop, D.J.J. Farnell, and C. Zeng, Phys. Rev. B 59, 1000 (1999).

9. R.F. Bishop, D.J.J. Farnell, S.E. Krüger, J.B. Parkinson, J. Richter, and C. Zeng, J. Phys.: Condens. Matter 12, 6887 (2000).

10. S.E. Krüger, J. Richter, J. Schulenburg, D.J.J. Farnell, and R.F. Bishop, Phys. Rev. $B$ 61, 14607 (2000).

11. S.E. Krüger and J. Richter, Phys. Rev. B 64, 024433 (2001).

12. J. Richter, S.E. Krüger, D.J.J. Farnell, and R.F. Bishop, in 150 Years of Quantum Many-Body Theory: A Festschrift in Honour of the 65th Birthdays of John W. Clark, 
Alpo J. Kallio, Manfred L. Ristig, and S. Rosati, edited by R.F. Bishop, K.A. Gernoth, and N.R. Walet, Series on Advances in Quantum Many-Body Theory Vol. 5 (World Scientific, Singapore, 2001), p. 239.

13. D.J.J. Farnell, R.F. Bishop, and K.A. Gernoth, Phys. Rev. B 63, 220402(R) (2001).

14. R.F. Bishop, Y. Xian, and C. Zeng, Int. J. Quantum Chem. 55, 181 (1995).

15. R.R.P. Singh, M.P. Gelfand, and D.A. Huse, Phys. Rev. Lett. 61, 2484 (1988).

16. N.B. Ivanov, S.E. Krüger, and J. Richter, Phys. Rev. B 53, 2633 (1996).

17. A.W. Sandvik and D.J. Scalapino, Phys. Rev. Lett. 72, 2777 (1994).

18. J. Villain, J. Phys. C: Solid State 10, 4793 (1977).

19. C. Gros, W. Wenzel, and J. Richter, Europhys. Lett. 32, 747 (1995).

20. M. Troyer, M. Imada, and K. Ueda, J. Phys. Soc. Jpn. 66, 2957 (1997). 